\title{
Automated Crystallite Orientation \& Phase Mapping in the TEM: Diffraction Contrast in Virtual Bright/Dark Field images of Polycrystalline Copper
}

\author{
S. Rouvimov*, P. Moeck*, E. F. Rauch**, S. Nicolopoulos***, and W. Neumann**** \\ * Department of Physics, Portland State University, Portland, OR 97207-0751 \\ ** CNRS- Grenoble INP, BP 46101 rue de la Physique, 38402 Saint Martin d'Hères, France \\ *** NanoMEGAS SPRL, Saint Jean Molenbeek, Brussels, B-1080, Belgium \\ **** Department of Chemistry, University of Oregon, Eugene, Eugene OR 97401
}

An automated crystal orientation and phase mapping method in a TEM has recently been developed [1-4]. This technique is complimentary to the electron backscatter diffraction (EBSD) technique (also known as orientation imaging microscopy or the back-scatter Kikuchi diffraction method) in scanning electron microscopy (SEM). While the EBSD technique is limited by the size of the poly-crystalline grains (its spatial resolution is limited to 20 to $80 \mathrm{~nm}$ ) and is sensitive to the plastic deformation, damage or contamination of the crystal surfaces, the new electron diffraction spot based method is working efficiently for the mapping of crystallite phases and orientations of polycrystalline samples at significantly smaller length scales and is rather insensitive to the plastic deformation state and the surface of nanocrystals. It has also been demonstrated that the precession electron diffraction mode $[5,6]$ improves the reliability of this technique significantly and the so called " $180^{\circ}$ ambiguity" in the indexing of spot patterns from the zero order Laue zone can be reliably overcome [4].

A typical crystallite orientation map thin polycrystalline copper film is shown in Fig. 1. The samples were analyzed by the FEI Tecnai $\mathrm{G}^{2}$ ST field-emission microscope equipped with the ASTAR/DigiSTAR system of NanoMEGAS. Virtual dark field images of individual crystallites or crystallite areas are obtained with the ASTAR software by a simple (computer) prompt after a suitable "virtual aperture" has been set around a particular reflection spot in one of the automatically recorded electron diffraction patterns from that crystallite. The corresponding dark field map then shows the crystallite or crystallite areas with bright contrast together with all of the various sample areas that contribute to the diffracted intensity within the virtual aperture. The experimental electron diffraction spot patterns that form the basis for the crystallite mapping process can simply be displayed by clicking with the mouse on a crystallite in either the virtual bright field map or the crystallite orientation map. In the top row of Fig. 2, four such electron diffraction patterns are shown. Due to the comparatively small precession angle, resemblances of "dynamical diffraction contrast" should be present in the virtual bright field map, Fig. 1a, and the virtual dark field maps, bottom row of Fig. 2. This is indeed the case as marked by arrows in Fig. $1 \mathrm{a}$ and the virtual dark field image in the first column of the bottom row of Fig. 2. While thickness fringes (of classical diffraction contrast) are a sequence of bright and dark stripes at the edge of a crystallite in the virtual bright field image of the marked crystallite, the contrast is reversed in the corresponding virtual dark field image. This kind of quasi-classical diffraction contrast at low precession angles will have its usage in more thorough off-line analyses of polycrystalline materials. Large precession angles, on the other hand, tend to average such contrasts out [7].

References

[1] E. Rauch et al., Microsc. Analysis, Issue 93, November 2008, S5. 
[2] P. Moeck at al., In: Electron Crystallography for Materials Research and Quantitative

Characterization of Nanostructured Materials, Mater. Res. Soc. Symp. Proc. 1184 (2009) 49.

[3] S. Rouvimov et al., Proc. 2009 NSTI, Vol. I (2009) 421.

[4] E. F. Rauch at al., Zeits. Krist. 225 (2010) 103.

[5] R. Vincent and P. Midgley, Ultramicroscopy 53 (1994) 271.

[6] P. Moeck and S. Rouvimov, In: Drug Delivery Nanoparticles: Formulation and

Characterization, Informa, New York, 2009, pp. 270-313.

[7] The Portland State University bases authors were supported by grants from the Oregon Nanoscience and Microtechnologies Institute and the Army Research Laboratories.
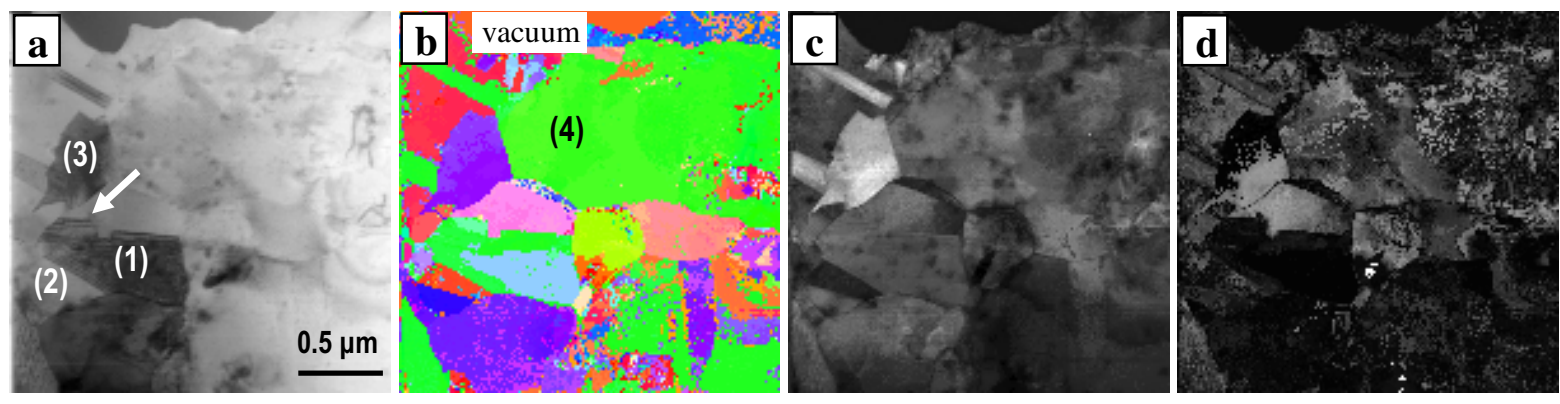

FIG. 1 Crystallite orientation mapping of polycrystalline copper, $0.15^{\circ}$ precession angle. (a) Virtual bright field map, (b) crystallite orientation map, (c) correlation index map, and (d) reliability index map from the same sample area.
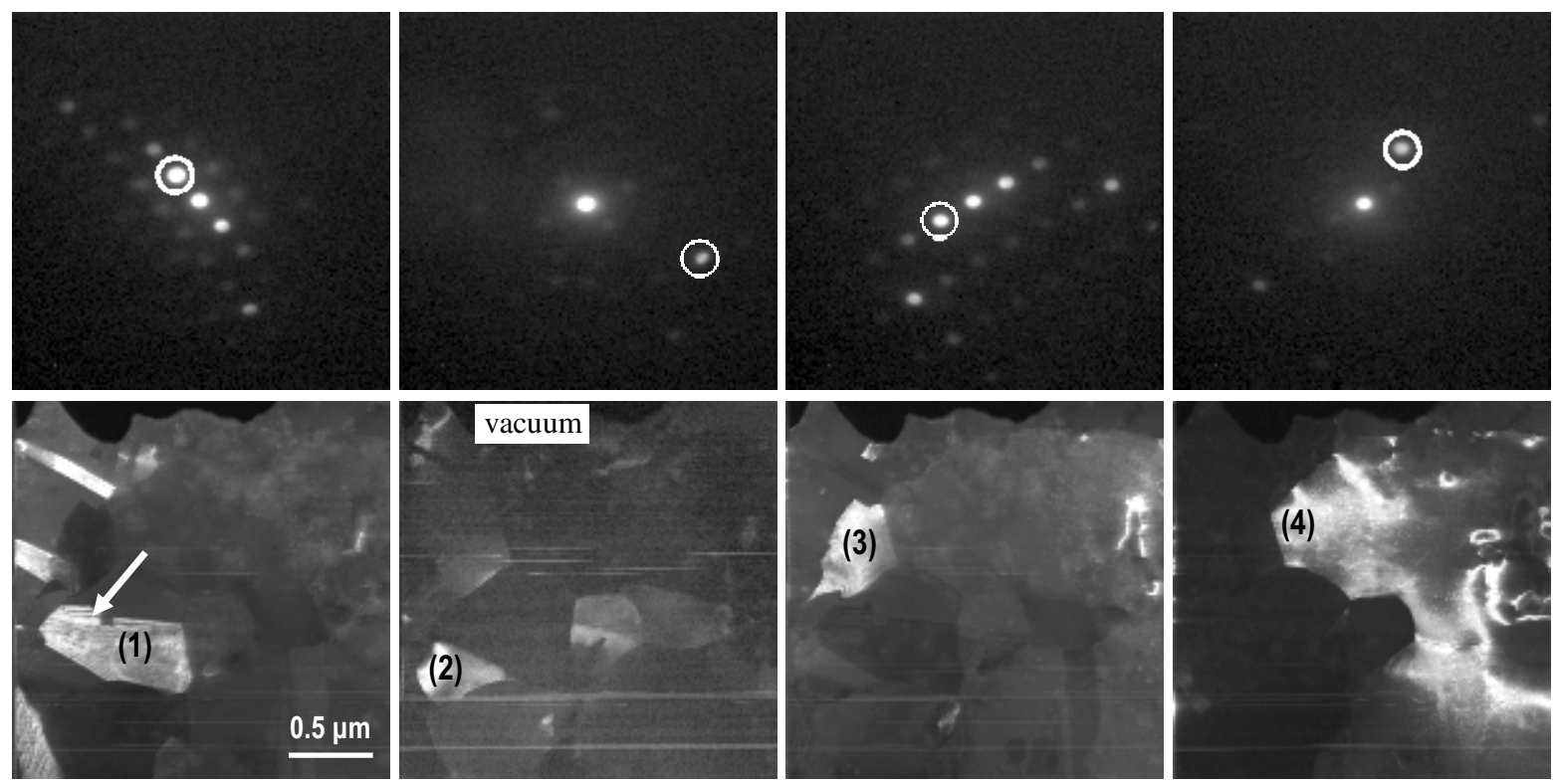

FIG. 2 Crystallite orientation mapping of polycrystalline copper, continuation from Fig. 1. Top row: Experimental diffraction spot patterns of selected crystallites (that appear brightest and largest in the bottom row). The positions of a 5 pixel diameter aperture that was imposed as mask on the diffraction spot patterns in order to create the dark field images (in the bottom row) are indicated by circles. Bottom row: Corresponding virtual dark field maps. 